\title{
Caractérisation de l'Emmental «Grand-Cru» français. I. Composition physico-chimique
}

\author{
JL Berdagué 1*, R Grappin 1, A Delacroix-Buchet ${ }^{1}$, B Chaillet ${ }^{2}$ \\ avec la collaboration technique de P Pidoux ${ }^{2}$ \\ 1 INRA station de recherches en technologie et analyses laitières, 39800 Poligny; \\ ${ }^{2}$ Institut technique du gruyère, 70240 Rioz, France
}

(Reçu le 11 octobre 1988; accepté le 20 septembre 1989)

\begin{abstract}
Résumé - L'Emmental "Grand-Cru» français est un produit dont la fabrication est soumise à une série de contraintes incluant l'ensemble de la chaîne de production. Ces contraintes interviennent au niveau de la production des laits (absence d'ensilage), de leur transformation (fabrication au plus tard $36 \mathrm{~h}$ après la première traite, absence de thermisation) et de l'affinage (10 semaines minimum). L'essai de caractérisation analytique de l'Emmental "Grand-Cru" français montre que ce fromage se distingue tout au long de l'année par un profil fermentaire typiquement propionique, une lipolyse réduite et une protéolyse plus intense que le fromage d'Emmental fabriqué avec du lait thermisé.
\end{abstract}

\section{fromage d'Emmental / composition / caractérisation analytique}

Summary - Characterisation of French Emmental "Grand-Cru». I. Physico-chemical composition. The production of French Emmental "Grand-Cru" manufactured in the Central Eastern part of France, is submitted to strict regulations with regard to milk production (no silage and cold storage of milk for less than $24 \mathrm{~h}$ ), milk processing (no heat treatment of the milk) and ripening conditions (over 10 weeks).

A comprehensive physico-chemical analytical study of 54 mature cheeses performed over a period of 1 year in 18 different cheese factories, located all over the areas of Emmental production, has shown that it is possible to characterize the Emmental "Grand-Cru" through its chemical composition. Compared to the other Emmental cheeses, the "Grand-Cru" cheeses have a lower amount of butyric acid and a higher level of acetic and isovaleric acids. Proteolysis, measured by the watersoluble and non-protein nitrogen fractions, is more important in the Emmental "Grand-Cru".

\section{Emmental cheese / composition / analytical characterisation}

\footnotetext{
*Adresse actuelle : INRA, Station de Recherches sur la Viande, Theix, 63122 Ceyrat, France.
} 


\section{INTRODUCTION}

Pendant plusieurs siècles, seul l'cEstCentral» (Centre-Est de la France) fut le lieu privilégié des fruitières, qui, outre le fromage de Comté, Appellation d'Origine, fabriquaient de l'Emmental. Dans les années 1970, la bonne valorisation du lait a incité les autres régions à développer la production de ce fromage sur l'ensemble du territoire national. Des usines de grande capacité ont alors fabriqué de l'Emmental et dans un souci de protection des transformations régionales, les professionnels de l'Est ont mis en place l'Emmental Est-Central "Grand-Cru».

Cet Emmental est aujourd'hui un produit dont la fabrication est soumise à une série de contraintes incluses dans le Label Agricole déposé par le Syndicat des Fabricants et Affineurs d'Emmental Traditionnel "Grand-Cru" (JO 180 NC du 5 août 1979). Ainsi, le lait utilisé pour la fabrication provient d'exploitations portant une attention particulière à l'état sanitaire et à l'hygiène des animaux. L'alimentation doit être exempte de produits d'ensilage et d'aliments fermentés. Le lait doit parvenir à l'atelier pour que l'emprésurage intervienne dans un délai maximum de $24 \mathrm{~h}$ (voire exceptionnellement $36 \mathrm{~h}$ en période froide), après la traite la plus ancienne. La fabrication de ce type d'Emmental ne peut s'exercer que dans un atelier recevant des laits de la zone de production et ne pratiquant pas la thermisation, opération qui consiste à chauffer le lait entre 61 et $63^{\circ} \mathrm{C}$ environ $30 \mathrm{~s}$.

La durée d'affinage de l'Emmental "Grand-Cru" doit être au moins égale à 10 semaines. Affinés, seuls les fromages répondant à certains critères de forme, de croûtage, de pâte, de goût, d'odeur et d'ouverture, se verront attribuer la dénomination d'Emmental Français Est-Central "Grand-Cru".
Face à ce type de produit, existent d'autres fabrications d'Emmental obtenues à partir de lait thermisé. Ces fromages sont fabriqués à partir de laits produits sans interdiction d'utilisation d'ensilage pour l'alimentation des animaux. La durée de stockage à basse température de ces laits est comprise entre 24 et $72 \mathrm{~h}$ et la durée d'affinage est réglementée ( 8 semaines au minimum).

L'objectif de cette étude est de caractériser le fromage d'Emmental Est-Central "Grand-Cru" par rapport à l'Emmental thermisé, à l'aide d'analyses physicochimiques. Cette caractérisation est effectuée à partir de fromages affinés fabriqués à différentes périodes de l'année, pour typer le produit indépendamment des fluctuations saisonnières de composition.

\section{MATÉRIEL ET MÉTHODES}

\section{Protocole expérimental}

Pour caractériser l'Emmental Est-Central "Grand-Cru", 3 groupes distincts de fromages ont été constitués :

- groupe 1 : Emmental Est-Central «GrandCru " fabriqué au lait cru (productions originaires des départements du Doubs et de la HauteSaône);

- groupe 2 : Emmental Franc-Comtois fabriqué avec du lait thermisé (productions originaires des départements du Doubs, de la Haute-Saône et du Territoire de Belfort);

- groupe 3 : Emmental Français d'autres provenances fabriqué aussi avec du lait thermisé.

Dans chacun des groupes, nous avons analysé 18 fromages prêts à être commercialisés, prélevés directement sur les sites d'affinage. Les fabrications de fromage ont été réparties sur 6 mois : mars, avril, mai, août, septembre et octobre. Le choix des entreprises de fabrication correspond à 3 tirages mensuels au hasard et sans remise durant 6 mois parmi les entreprises 
des groupes concernés. Au total $\mathbf{5 4}$ fromages ont été analysés représentant 54 fabrications différentes.

L'ensemble des analyses a été effectué sur des fromages affinés, c'est-à-dire n'ayant pas obligatoirement le même âge, mais représentatifs du produit lors de sa commercialisation. Pour chaque fromage les analyses ont été faites sur la partie centrale de la meule après élimination de 2 centimètres sous la croûte.

\section{Techniques analytiques}

\section{Analyses physico-chimiques classi- ques}

Une série d'analyses donnant la répartition pondérale des principaux constituants du fromage a permis de mesurer :

- l'extrait sec (normes FIL-IDF : 4A, 1982);

- l'azote total (normes FIL-IDF : 20A, 1986);

- la matière grasse (méthode butyrométrique de Heiss, 1961);

- le calcium total (Pearce, 1977);

- les chlorures, exprimés en chlorure de sodium (Booy, 1968).

- les lactates $L$ et $D$ (méthode enzymatique Boehringer-Mannheim, réf. 139-084);

- le pH (électrode combinée, placée 30 s en contact avec un broyat de fromage tassé dans un godet).

\section{Analyse des acides gras libres}

Les acides gras libres (AGL) ont été extraits et analysés dans les conditions suivantes :

- pour les acides gras volatils (AGV), la méthode utilisée est l'adaptation au fromage par Agnet (1979) d'une technique mise au point par Kuzdzal-Savoie et Kuzdzal (1967), permettant le dosage des $A G V$ de $\mathrm{C}_{2}$ à $\mathrm{C}_{6}$ inclus.

- pour les AGL à plus de 6 atomes de carbone l'extraction consiste à broyer $10 \mathrm{~g}$ de fromage acidifiés par $2 \mathrm{ml}$ d'acide sulfurique à 10\% (v/v) dans $40 \mathrm{ml}$ d'un mélange de diéthyl-oxyde et d'éther de pétrole (50/50 v/v) après addition de $80 \mathrm{mg}$ d'étalon interne (acide tridécanoïque).

Le broyat est centrifugé 2 min à $1500 \mathrm{~g}$ et la phase éthérée surnageante est récupérée. Le culot est extrait une seconde fois par $40 \mathrm{ml}$ du mélange d'éthers. Les 2 phases éthérées ainsi obtenues contiennent la majeure partie des graisses et des AGL du fromage. Les AGL sont séparés des graisses (Kuzdzal-Savoie, Kuzdzal, 1967) et stockés sous forme de savons.

Pour l'analyse chromatographique, les savons sont acidifiés dans des tubes à vis par $1 \mathrm{ml}$ d'une solution aqueuse de $\mathrm{HCl}(50 / 50$, v/v), en présence de $1 \mathrm{ml}$ d'hexane. Après blocage de la phase aqueuse par une quantité suffisante de sulfate de sodium anhydre, la phase hexane contenant les AGL est prête à être injectée dans le chromatographe (volume injecté : $2 \mu \mathrm{l}$ ). La chromatographie en phase gazeuse est réalisée selon les conditions décrites par Supelco (1975), sur un matériel Intersmat 121 DFL.

\section{Analyse des fractions azotées}

L'azote soluble ou azote non caséine (NS) et l'azote non protéique (NPN) ont été fractionnés d'après Lenoir (1963) et dosés selon la norme FIL-IDF (1986). Le dosage de l'ammoniaque est effectué selon la méthode d'Amariglio (1986) adaptée à $5 \mathrm{~g}$ de fromage.

Fractions caséines : les séparations ont été effectuées par électrophorèse sur gel d'acrylamide-bisacrylamide-agarose, dans les conditions décrites par Collin et al (1987). Les fractions $\alpha S_{1}, \alpha S_{1} l, \beta, \beta$ dégradée, $\gamma_{1}, \gamma_{2}$ et $\gamma_{3}$ ont été étudiées et les résultats exprimés en pourcentage de l'aire totale des caséines étudiées.

Fraction amino-peptidasique : la séparation des constituants de la fraction azotée soluble dans l'eau, préparée selon la technique de Kuchroo et Fox (1982a, b), a été réalisée par chromatographie en phase liquide selon Aston et Creamer (1986), à l'aide d'un matériel Varian 5020 équipé d'un détecteur UV variable réglé à $214 \mathrm{~nm}$.

\section{Analyses statistiques}

\section{Statistiques élémentaires}

La moyenne et l'écart type ont été calculés pour toutes les variables, sur l'ensemble des individus et pour chacun des groupes définis. 


\section{Relations entre variables}

Pour certaines relations importantes, nous effectuerons une série de régressions linéaires simples.

\section{Etude des groupes d'individus}

L'incidence du groupe d'appartenance des différents fromages sur l'ensemble des variables a été quantifiée par une analyse de la variance à une voie, en randomisation totale selon le modèle mathématique :

$$
Y_{i, j}=\mu+\alpha_{i}+R_{i, j}
$$

avec $Y_{i, j}=$ valeur de la caractéristique du fromage; $\mu=$ terme constant à tous les fromages; $\alpha_{i}=$ effet moyen du facteur groupe d'appartenance; $i=$ nombre de niveaux du facteur $(i=3)$; $j=$ nombre de répétitions $(j=18) ; R_{i, j}=$ terme aléatoire dans le modèle.

Lorsque l'effet du groupe d'appartenance est significatif, une comparaison des moyennes a été réalisée par le test de Newmann-Keuls.

Une analyse discriminante pas à pas a été effectuée à partir des variables les plus indépendantes pour séparer les fromages du groupe 1 de l'ensemble des autres fromages. L'ordre d'introduction des variables dans le calcul dépendait du $F$ de Fisher, calculé entre les groupes. L'introduction des variables dans l'analyse discriminante a été stoppée lorsque le pourcentage d'individus affectés correctement après calcul, dans leur groupe d'appartenance (fromages des groupes 1 ou 2 et 3 réunis) n'augmentait plus.

Pour comparer des fromages d'âge identique, nous avons considéré la sous-population de 19 fromages âgés de 11 semaines, composée de 9 emmentals du groupe 1 et de 10 emmentals des groupes 2 et 3 .

\section{RÉSULTATS}

L'âge moyen de chacun des 3 groupes de fromages que nous étudions est de $83 \pm 9$ jours pour le groupe $1 ; 69 \pm 16$ jours pour le groupe 2 et $62 \pm 11$ jours pour le groupe 3 .
Analyses physico-chimiques (tableau IA)

La teneur en sel des fromages des groupes 1 et $2(0,42 \mathrm{~g}$ et 0,40 par $100 \mathrm{~g})$ est inférieure à celle des fromages du groupe $3(0,55 \mathrm{~g}$ par $100 \mathrm{~g})$. Les valeurs moyennes d'extrait $\sec (63,13 \%)$, de matières grasses $(29,90 \%)$, d'azote total $(28,95 \%)$, de calcium $(981 \pm 40 \mathrm{mg}$ par $100 \mathrm{~g})$ et de $\mathrm{pH}(5,71)$ ne sont en revanche pas significativement différentes entre les 3 groupes considérés.

\section{Analyse des lactates et des acides gras libres (tableau IB)}

La teneur en acide butyrique des fromages du groupe 1 (14 mg par $100 \mathrm{~g}$ ) est beaucoup plus faible que celles des fromages des groupes 2 et 3 (respectivement 40 et $59 \mathrm{mg}$ par $100 \mathrm{~g}$ ). Inversement, dans le groupe 1 les teneurs sont plus élevées en acides acétique et isovalérique (respectivement 370 et $3,5 \mathrm{mg}$ pour $100 \mathrm{~g}$ ). Les dosages de l'acide propionique et des isomères optiques des lactates ne révèlent pas de différence significative entre les groupes. La teneur moyenne en acide propionique de tous les emmentals est de $284 \mathrm{mg}$ pour $100 \mathrm{~g}$ de fromage, celle en lactates totaux résiduels de $789 \mathrm{mg}$ pour $100 \mathrm{~g}$.

Pour les chaînes carbonées plus longues $\left(\mathrm{C}_{8}: 0\right.$ à $\left.\mathrm{C}_{18}: 3\right)$, seuls les acides laurique $\left(\mathrm{C}_{12}: 0\right)$ et linolénique $\left(\mathrm{C}_{18}: 3\right)$ permettent de distinguer (tableau IB) les fromages Franc-Comtois (groupes 1 et 2) des fromages du groupe 3 . Les emmentals des groupes 1 et 2 ont les teneurs les plus faibles en acides laurique (15,2 et $17,6 \mathrm{mg}$ contre $21,3 \mathrm{mg}$ pour $100 \mathrm{~g}$ ) et les teneurs les plus élevées en acide linolénique (environ 11,5 contre $7,3 \mathrm{mg}$ pour $100 \mathrm{~g})$. 
Les teneurs moyennes de l'Emmental en acides gras libres à 6 ou plus de 6 atomes de carbone (considérés habituellement d'origine lipolytique) : $424 \pm 97 \mathrm{mg}$ pour $100 \mathrm{~g}$ de fromage ou bien celles en acides gras libres totaux (issus des fermentations: $\mathrm{C}_{2}, \mathrm{C}_{3}, \mathrm{C}_{4}$; de la protéolyse : $\mathrm{iC}_{5}$ et de la lipolyse): $1087 \pm 194 \mathrm{mg}$ pour $100 \mathrm{~g}$ de fromage ne caractérisent pas les groupes d'Emmental.

Pour un âge identique des fromages; le tableau II montre que les emmentals des groupes 2 et 3 réunis ont des teneurs supérieures en AGL à plus de 6 carbones à celle de l'emmental Est-Central "GrandCrus (respectivement $497 \pm 95$ contre 375 $\pm 53 \mathrm{mg}$ par $100 \mathrm{~g}$ de fromage).

\section{Analyse des fractions azotées (tableau IC)}

Les fromages du groupe 1 se caractérisent nettement par les rapports NS/NT, NPN/ NT et les teneurs en ammoniaque les plus élevés. Malgré un affinage plus long, l'âge des fromages n'explique pas toutes les différences de protéolyse. Ainsi, dans l'exemple du rapport NS/NT, le calcul des équations de régression donnant la valeur de ce rapport en fonction de l'âge du fromage, montre que selon le type de fabrication (fromages du groupe 1 ou fromages des groupes 2 et 3 réunis) on obtient des droites distinctes correspondant à deux cinétiques de protéolyse (fig 1). Une obser-

Tableau IA. Influence de l'origine des fromages d'Emmental sur leur composition physico-chimique. Résultats de l'analyse de la variance.

Influence of the origin of Emmental cheeses on their physico-chemical composition. Analysis of variance results.

1
3

\begin{tabular}{lll}
\hline$G_{1}$ & $G_{2}$ & $G_{3}$
\end{tabular}

\begin{tabular}{llrcccc}
\hline Extrait sec & NS & $63,13 \pm 1,11$ & $63,34 \pm 1,23$ & $63,07 \pm 1,10$ & $62,98 \pm 0,96$ \\
Matière grasse & NS & $29,90 \pm 1,46$ & $29,83 \pm 1,61$ & $29,76 \pm 1,41$ & $30,10 \pm 1,33$ \\
Azote total & NS & $28,95 \pm 0,99$ & $29,14 \pm 0,82$ & $28,81 \pm 0,89$ & $28,88 \pm 1,07$ \\
pH & NS & $5,71 \pm 0,11$ & $5,73 \pm 0,11$ & $5,71 \pm 0,10$ & $5,69 \pm 0,12$ \\
Calcium & NS & $981,2 \pm 40,3$ & $979,4 \pm 4,01$ & $979,7 \pm 41,0$ & $984,4 \pm 39,7$ \\
Chlorure de sodium ${ }^{* *}$ & 17,1 & $0,46 \pm 0,16$ & $0,42 \pm 0,16^{a}$ & $0,40 \pm 0,12^{a}$ & $0,55 \pm 0,14^{b}$
\end{tabular}

$1=$ Test de $F$ : seuil de signification (NS = non significatif, ${ }^{*} P<0,05 ;{ }^{* *} P<0,01$ ) et coefficient de détermination exprimé en pourcentage de la variance totale

ANOVA F test $=$ level of significance (NS = non significant, $* P<0.05, * P<0.01$ ) and coefficient of determination expressed in \% (total variance)

2 = Moyenne générale, écart type (en $\mathrm{g} / 100 \mathrm{~g}$ )

Overall mean and standard deviation $(\mathrm{g} / 100 \mathrm{~g})$

3 = Moyennes et écart types (en g/100 g) des groupes : $G_{1}=$ Emmental Est-Central "Grand-Cru», $G_{2}:$ Emmental Franc-Comtois thermisé, $\mathrm{G}_{3}:$ Emmental d'autres provenances.

Means and standard-deviation of the groups: $G_{1}=$ Central-East Emmental "Grand Cru», $G_{2}=$ «Franche-Comté Emmental from heat treated milk; $G_{3}=$ Emmental from other regional.

Test de Newmann-Keuls : les groupes homogènes sont affectés d'une même lettre.

Newmann-Keuls test: groups with the same transcript do not differ $(P<0.05)$. 
vation identique peut être faite avec les différents indicateurs de la protéolyse, à l'exception des fractions $\gamma_{1}, \gamma_{2}, \gamma_{3}$ et $\beta$ dégradée qui sont mal corrélées à l'âge des fromages, probablement en raison de leurs fortes variations mensuelles. La comparaison directe des 2 populations d'âge identique confirme clairement que la protéolyse est plus intense à âge égal dans les fromages du groupe 1 (tableau II).

Trois pics de la fraction aminopeptidasique, communs à l'ensemble des chromatogrammes (fig 2) permettent de

Tableau IB. Influence de l'origine des fromages d'Emmental sur leur composition physico-chimique. Résultats de l'analyse de la variance.

Influence of the origin of Emmental cheeses on their composition. Analysis of variance results.

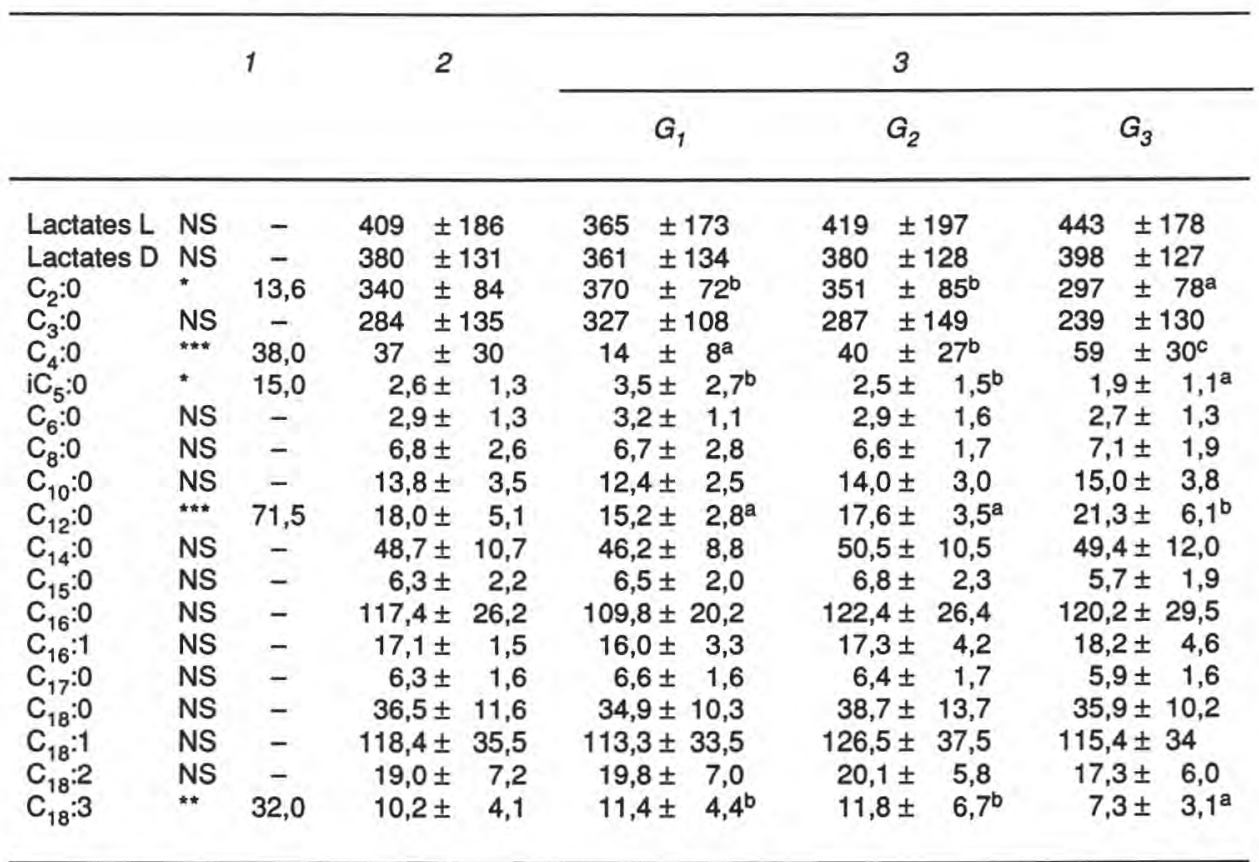

$1=$ Test de $F$ de l'analyse de la variance : seuil de signification (NS = non significatif, ${ }^{*}=P<0,05 ;{ }^{* *} P<0,01 ;{ }^{* * *} P$ $<0,001)$ et coefficient de détermination exprimé en pourcentage de la variance totale.

ANOVA F test: level of significance (NS = non significant, ${ }^{*} P<0.05,{ }^{*} P<0.01, \cdots P<0.001$ ) and coefficient of determination expressed in $\%$ of the total variance.

2 = Moyenne générale, écart type (en $\mathrm{mg} / 100 \mathrm{~g}$ )

Overall mean and standard deviation (in $\mathrm{mg} / 100 \mathrm{~g}$ )

3 = Moyennes et écart types (en mg/100 g) des groupes : $G_{1}=$ Emmental Est-Central "Grand-Cru", $G_{2}=E m m e n t a l$ Franc-Comtois thermise, $\mathrm{G}_{3}=$ Emmental d'autres provenances.

Means and standard-deviation of the groups: $G_{1}=$ Central East Emmental "Grand-Cru", $G_{2}=$ "Franche-Comte" Emmental from heat heated milk, $G_{3}=$ Emmental from other regions.

Test de Newmann-Keuls : les groupes homogènes sont affectés d'une même lettre.

Newmann-Keuls test: Groups with the same superscript do not differ $(P<0.05)$. 
distinguer significativement $(P<0,01)$ les groupes d'Emmental. Ces pics ont des temps de rétention correspondant à ceux de la tyrosine, de la phénylalanine et du tryptophane. Un nombre important d'autres pics apparaissent sur les chromatogrammes, mais l'information relative à leur surface n'a pas été exploitée. Leur mauvaise résolution et leur apparition (présence-absence) très variable selon les chromatogrammes, empêche de relier un pic précis à une surface intégrée au-delà d'un temps de rétention de $11 \mathrm{~min}$, zone correspondant aux peptides.

Tableau IC. Influence de l'origine des fromages d'Emmental sur leur composition physico-chimique. Résultats de l'analyse de la variance.

Influence of the origin of Emmental cheeses on their composition. Analysis of variance results.

1

2
3

$G_{2} \quad G_{3}$

Fractions solubles globales

$\begin{array}{llllrll}\text { NS/NT } & * * & 40,6 & 20,71 \pm 4,36 & 24,61 \pm 2,66^{\mathrm{b}} & 19,22 \pm 4,10^{\mathrm{a}} & 18,31 \pm 3,03^{\mathrm{a}} \\ \text { NPN/NT } & * * & 31,1 & 15,16 \pm 4,41 & 18,30 \pm 3,05^{\mathrm{c}} & 14,87 \pm 4,39^{\mathrm{b}} & 12,30 \pm 3,39^{\mathrm{a}} \\ \text { Ammoniaque } & * * & 24,8 & 83,83 \pm 32,55 & 101,58 \pm 25,02^{\mathrm{c}} & 87,15 \pm 33,56^{\mathrm{b}} & 62,75 \pm 25,60^{\mathrm{a}}\end{array}$

Fractions caséine

$\begin{array}{llrrrrrr}\alpha S_{1} & * * * & 41,6 & 16,37 \pm 5,97 & 11,11 \pm 3,67^{\mathrm{a}} & 17,47 \pm 5,79^{\mathrm{b}} & 20,44 \pm 3,92^{\mathrm{b}} \\ \alpha \mathrm{S}_{1} \mid & * * * & 38,5 & 30,70 \pm 4,93 & 35,03 \pm 3,80^{\mathrm{b}} & 29,05 \pm 4,15^{\mathrm{a}} & 28,04 \pm 3,73^{\mathrm{a}} \\ \beta & * * & 19,0 & 25,08 \pm 4,10 & 22,70 \pm 2,63^{\mathrm{a}} & 26,93 \pm 3,93^{\mathrm{b}} & 25,65 \pm 4,12^{\mathrm{b}} \\ \beta \text { dég. } & \text { NS } & 6,2 & 6,80 \pm 2,12 & 6,80 \pm 2,30 & 6,90 \pm 2,1 & 6,03 \pm 2,2 \\ \gamma_{1} & * * & 12,3 & 7,31 \pm 1,74 & 8,16 \pm 1,47^{\mathrm{b}} & 7,03 \pm 1,88^{\mathrm{a}} & 6,72 \pm 1,30^{\mathrm{a}} \\ \gamma_{2} & * * & 20,3 & 7,64 \pm 1,99 & 8,92 \pm 1,68^{\mathrm{b}} & 7,09 \pm 1,63^{\mathrm{a}} & 6,92 \pm 2,00^{\mathrm{a}} \\ \gamma_{3} & * * * & 29,5 & 5,96 \pm 1,79 & 7,30 \pm 1,40^{\mathrm{b}} & 5,60 \pm 1,45^{\mathrm{a}} & 6,20 \pm 2,10^{\mathrm{a}}\end{array}$

Fractions aminopeptidasiques

\begin{tabular}{llllrll} 
Tyrosine $* *$ & 18,4 & $853 \pm 309$ & $1066 \pm 194^{\mathrm{c}}$ & $827 \pm 327^{\mathrm{b}}$ & $665 \pm 246^{\mathrm{a}}$ \\
Phénlylalanine *** & 28,4 & $641 \pm 233$ & $732 \pm 225^{\mathrm{b}}$ & $602 \pm 104^{\mathrm{a}}$ & $590 \pm 150^{\mathrm{a}}$ \\
Tryptophane ** & 16,1 & $474 \pm 283$ & $605 \pm 180^{\mathrm{b}}$ & $456 \pm 260^{\mathrm{a}}$ & $360 \pm 210^{\mathrm{a}}$ \\
\hline
\end{tabular}

$1=$ Test de $F$ de l'analyse de la variance : seuil de signification (NS = non significatif, $* P<0,05 ;{ }^{* * *} P<0,01 ; * * * P<$ 0,001 ) et coefficient de détermination exprimé en pourcentage de la variance totale.

ANOVA F test: level of significance ( $N S=$ non significant, ${ }^{*} P<0.05,{ }^{* *} P<0.01,{ }^{* * *} P<0.001$ ) and coefficient of determination expressed in \% of the total variance.

2 = Moyenne générale, écart type (ammoniaque : $\mathrm{mg} / 100 \mathrm{~g}$, fraction amino-peptidasique : unités arbitraires de surface, autres composés : sans unité).

Overall mean and standard-deviation (ammonia $=m g / 100 \mathrm{~g}$, amino-peptidase fraction: area in arbitrary units, other compounds: no unit).

$3=$ Moyennes et écart types (ammoniaque : $\mathrm{mg} / 100 \mathrm{~g}$, fraction amino-peptidastique : unités arbitraires de surface, autres composés : sans unité) des groupes : $G_{1}$ : Emmental Est-Central "Grand-Cru", $G_{2}$ : Emmental FrancComtois thermisé, $\mathrm{G}_{3}$ : Emmental d'autres provenances.

Means and standard-deviation the groups: $G_{1}=$ Central-East Emmental "Grand-Cru», $G_{2}=$ "Franche-Comte $* E m$ mental from heat treated milk, $G_{3}=$ Emmental from other regions.

Test de Newmann-Keuls : les groupes homogènes sont affectés d'une même lettre.

Newmann-Keuls test: groups with the same superscript do not differ $(P<0.05)$. 


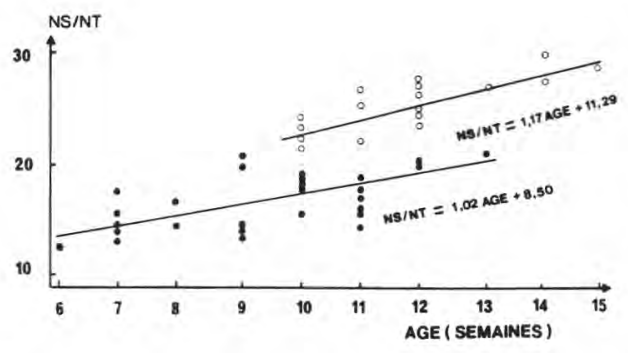

Fig 1. Evolution du rapport NS/NT en fonction de l'âge des fromages dans le cas des Emmentals du groupe 1 (Emmental Est-Central "Grand-Cru" : O) ou des fromages des groupes 2 et 3 (Emmental «Franc-Comtois thermisé" et Emmental "autres provenances" :

Variation of the ratio NS/NT (non casein soluble nitrogen/total nitrogen) with the age of the Emmental cheese: O: Central East Emmental "Grand-Cru" (Group 1) : "Franche-Comté" Emmental from heat treated milk and Emmental from other regions (Groups 2 and 3).

Les 3 pics étudiés présentent une surface plus importante dans le cas des fromages du groupe 1 (tableau IC). D'une manière générale, la surface totale du chromatogramme est plus importante pour les fromages de ce groupe. Cependant, aucun pic n'a permis de caractériser la technologie de fabrication associée aux fromages d'Emmental Est-Central «GrandCru».

L'analyse discriminante montre que les 4 variables initiales qui offrent le meilleur éclatement des groupes sont, par ordre d'importance : le rapport NS/NT, et les teneurs en acide butyrique $\left(\mathrm{C}_{4}\right)$, isovalérique $\left(\mathrm{iC}_{5}\right)$ et acétique $\left(\mathrm{C}_{2}\right)$ (fig 3 ). Lors de l'introduction successive dans le calcul des 4 variables dans cet ordre, le pourcentage d'individus bien classés selon la variable discriminante évolue de 62, 89, 92 à $94 \%$. Les fromages mal classés correspondent exclusivement à des fromages des groupes 2 et 3 , n'ayant pas présenté de fermentation butyrique $\left(C_{4} \leq 14 \mathrm{mg}\right.$ pour $100 \mathrm{~g}$ de fromage), plus protéolysés que la moyenne de leur groupe $(22,4<\mathrm{NS} / \mathrm{NT}<$ 29,2 ) et affinés entre 84 et 105 jours. La stabilité de l'éclatement ainsi obtenu a été confirmée à partir d'une population test supplémentaire de 20 emmentals : 10 fabriqués à partir de lait cru et 10 fabriqués à partir de lait thermisé.

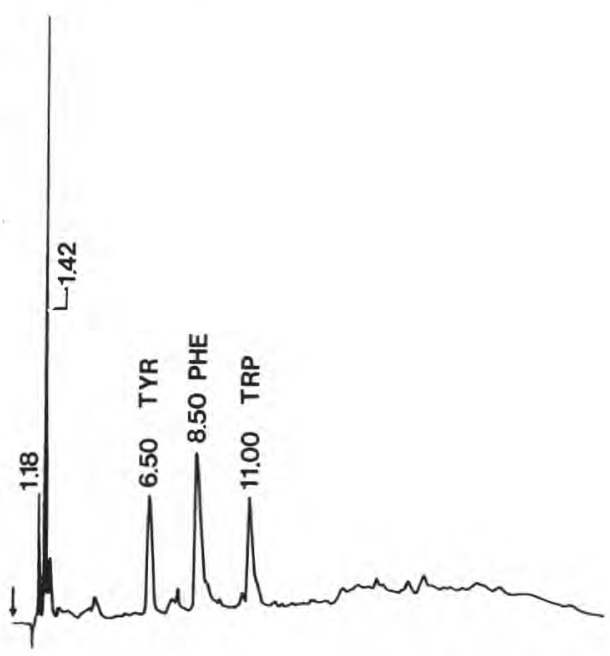

Fig 2. Exemple de séparation par chromatographie liquide de la fraction aminopeptidasique. Les temps de rétention (min) des pics les plus importants sont notés sur le chromatrogramme $(T Y R=$ tyrosine, $\mathrm{PHE}=$ phénylalanine, $\mathrm{TRP}=$ tryptophane).

Example of HPLC chromatogram of the aminopeptidase fraction. Rétention time (min) of the most important peaks are characterized (TYR = tyrosin; $P H E=$ phenylalanine; TRP = tryptophan). 
Tableau II. Comparaison de la composition, en acides gras libres, en fractions azotées (NS et NPN) et en fractions caséines de 2 populations de fromages d'Emmental affinés de 11 semaines.

Free fatty acids, Nitrogen fractions (NS = non casein soluble nitrogen; NPN = non protein nitrogen) and casein fraction contents of 2 populations of 11-week-old Emmental cheeses.

1

Lipolyse : acides gras libres $(\mathrm{mg} / 100 \mathrm{~g})$

$$
\begin{aligned}
& C_{8}: 0 \\
& C_{10}: 0 \\
& C_{12}: 0 \\
& C_{14}: 0 \\
& C_{15}: 0 \\
& C_{16}: 0 \\
& C_{16}: 1 \\
& C_{17}: 0 \\
& C_{18}: 0 \\
& C_{18}: 1 \\
& C_{18}: 2 \\
& C_{18}: 3
\end{aligned}
$$

$\begin{array}{rlr}5,8 & \pm & 1,2 \\ 12,2 & \pm & 2,3 \\ 14,9 & \pm & 2,9 \\ 43,8 & \pm & 6,5 \\ 5,9 & \pm & 1,6 \\ 106,4 & \pm & 14,2 \\ 15,1 & \pm & 1,8 \\ 6,0 & \pm & 0,9 \\ 30,5 & \pm & 6,9 \\ 103,4 & \pm & 22,5 \\ 17,1 & \pm & 4,0 \\ 9,7 & \pm & 4,0\end{array}$

$\begin{array}{rlr}7,8 & \pm & 2,1 \\ 16,1 & \pm & 3,6 \\ 19,9 & \pm & 4,0 \\ 54,5 & \pm & 10,8 \\ 7,5 & \pm & 2,0 \\ 131,0 & \pm & 25,8 \\ 19,3 & \pm & 3,3 \\ 7,4 & \pm & 1,6 \\ 44,7 & \pm & 12,8 \\ 148,2 & \pm & 33,7 \\ 22,9 & \pm & 6,1 \\ 14,4 & \pm & 6,1\end{array}$

Protéolyse : fractions azotées (pourcentage pondéral) ammoniac (mg/100 g), caséines (pourcentage de la surface totale)

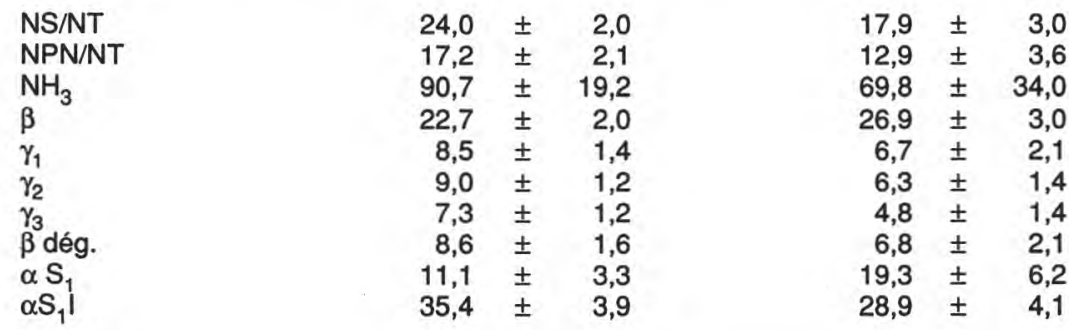

1 = Emmentals Est-Central "Grand-cru» (9 fromages du groupe 1).

Central-East Emmental "Grand-Cru" (9 cheeses from group 1).

$2=$ Emmentals d'autres fabrications (10 fromages des groupes 2 et 3 ).

Emmental of other origins (10 cheeses from groups 2 and 3 ).

\section{DISCUSSION}

L'étude des profils fermentaires montre que les fromages du groupe 1 se caractérisent par une teneur faible en acide butyrique, probablement d'origine lipolytique, voisine de celle observée dans le fromage de Comté (Berdagué et al, 1987). En revanche, les teneurs élevées de cet acide dans les fromages des groupes 2 et 3 proviennent, pour l'essentiel, de la fermentation butyrique. L'utilisation d'ensilage dans l'alimentation des animaux, en augmentant le risque de contamination des laits puis des fromages par Clostridium tyrobutyricum (FNLP-ITG, 1982), est vraisemblablement à l'origine de cette fermentation. 


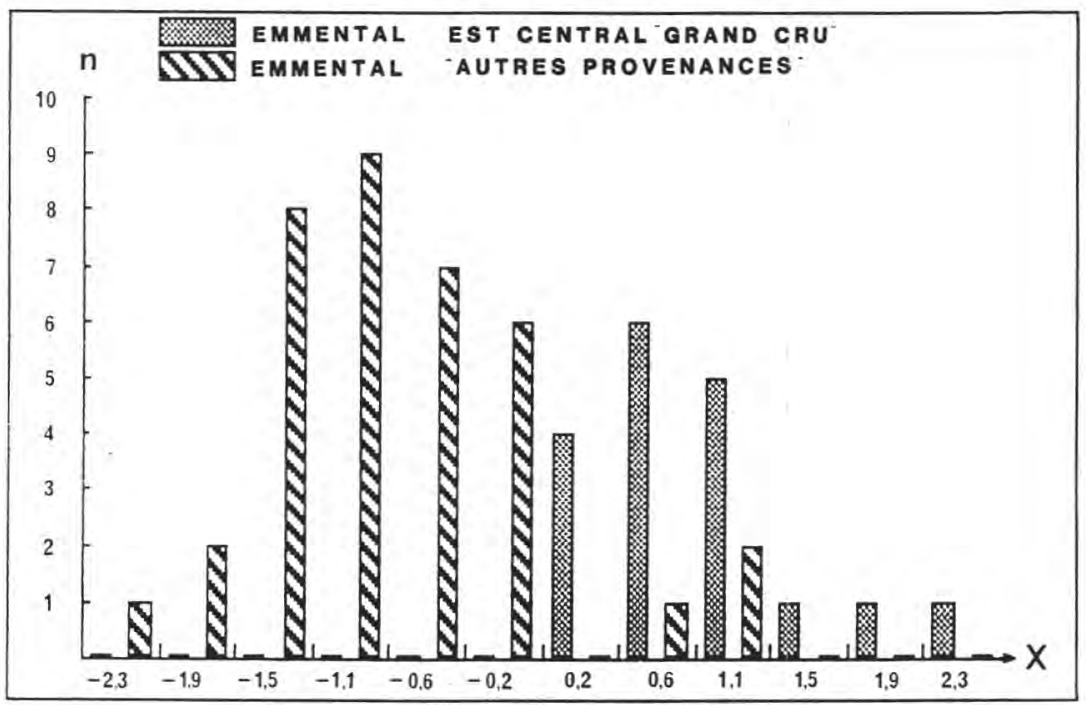

Fig 3. Histogramme des deux populations d'Emmental en fonction de la variable discriminante $(X)$. $X=14,48 \times 10^{-2} \mathrm{NS} / \mathrm{NT}-12,10 \times 10^{-3} \mathrm{C}_{4}+16,72 \times 10^{-2} \mathrm{iC}_{5}-2,71 \times 10^{-4} \mathrm{C}_{2}-2,90$ (NS/NT = azote soluble/azote total; $\mathrm{C}_{2}, \mathrm{C}_{4}, \mathrm{iC}_{5}=$ acides acétique, butyrique et isovalérique). Frequency histogram of 2 populations of Emmental cheeses, according the variable $\mathrm{X}=14.48 \times 10^{-2} \mathrm{NS} / \mathrm{NT}-12.10 \times 10^{-3} \mathrm{C}_{4}+16.72 \times 10^{-2} \mathrm{iC}_{5}-2.71 \times 10^{-4} \mathrm{C}_{2}-2.90$ (NS/NT = non casein soluble nitrogen/total nitrogen; $C_{2}, C_{4}, i C_{5}=$ acetic butyric and isovaleric acids).

Aucune différence d'intensité de la fermentation propionique n'est, en retour, observée à partir du dosage des lactates résiduels et de l'acide propionique sur fromages affinés (tableau IB). Les différences de teneur en acide acétique, qui distinguent les fromages Franc-Comtois (groupes 1 et 2) des fromages d'autres provenances, peuvent être d'origine hétérofermentaire, mais aussi provenir du catabolisme des acides aminés car le classement des teneurs moyennes en acide acétique des trois groupes de fromages (tableau IB) concorde assez bien avec celui des différents indicateurs de la protéolyse (tableau IC).

Les teneurs en acides gras libres (tableau IB) correspondent au turn-over des acides gras issus d'une part de la lipolyse (Kuzdzal-Savoie et Day, 1966) ou de la dégradation des acides gras dans le fromage (Dumont et Adda, 1978) et d'autre part des synthèses microbiennes. Les différences de composition en acide laurique $\left(C_{12: 0}\right)$ et linolénique $\left(C_{18: 3}\right)$ semblent être un phénomène régional, puisque les fromages Franc-Comtois (groupes 1 et 2) se distinguent des fromages "autres provenances".

La composition moyenne des 54 fromages en AGL à plus de 6 atomes de carbone est très voisine de celle observée dans l'Emmental Suisse par Langler et Day (1966) et le pourcentage pondéral de chacun de ces AGL est voisin de celui mesuré dans les acides gras totaux du lait 
(tableau III). L'hydrolyse globale des triglycérides dans les fromages est en définitive assez peu spécifique de la longueur de la chaîne carbonée des acides gras sur le glycéride, ce qui confirme plusieurs résultats antérieurs (De Man, 1966; Choisy et al, 1987). La faible lipolyse mesurée dans l'Emmental $(0,42 \mathrm{~g}$ d'AGL d'origine lipolytique pour $29,90 \mathrm{~g}$ de matières grasses dans $100 \mathrm{~g}$ de fromage) est une caractéristique générale des fromages à pâte pressée cuite. On note cependant un pourcentage moyen plus élevé d'acides gras libres insaturés $\left(\mathrm{C}_{16: 1}, \mathrm{C}_{18: 2}\right.$ et $\left.\mathrm{C}_{18: 3}\right)$ dans le fromage d'Emmental, que dans les acides gras totaux du lait. Ces acides gras insaturés peuvent provenir des synthèses microbiennes.

Le stockage limité des laits dans le cas de l'Emmental Est-Central "Grand-Cru» pourrait être un des facteurs à considérer pour expliquer la lipolyse plus réduite dans ces fromages (tableau II). En effet, certaines souches de Pseudomonas de la flore psychrotrophe peuvent se développer durant le stockage du lait à basse température et synthétiser des lipases thermostables (Cogan, 1977) actives pendant l'affinage (Driessen et Stadhouders, 1975; Driessen, 1983).

Tableau III. Comparaison de la composition moyenne en acides gras libres des $\mathbf{5 4}$ fromages d'Emmental, à celle du fromage d'Emmental suisse (résultats exprimés en $\mathrm{mg}$ par $100 \mathrm{~g}$ de fromage) et comparaison du pourcentage pondéral des différents acides gras libres du fromage au pourcentage pondéral des acides gras totaux du lait.

Comparison of the average contents of free fatty acids between the 54 French Emmental cheeses and Swiss Emmental cheese (results expressed in $\mathrm{mg} / 100 \mathrm{~g}$ cheese) and comparison by weight between the percentage of free fatty acids in the cheeses and the percentage of milk fatty acids.

\begin{tabular}{|c|c|c|c|c|c|}
\hline & & & & & 3 \\
\hline & & & $(\mathrm{mg} / 100 \mathrm{~g})$ & $\begin{array}{l}\text { (proportions } \\
\text { pondérales) }\end{array}$ & $\begin{array}{c}\text { (proportions } \\
\text { pondérales) }\end{array}$ \\
\hline $\mathrm{C} 4: 0$ & - & - & - & - & 1,4 \\
\hline C6:0 & 11,5 & 4,1 & $3,1+1,1$ & $0,7+0,3$ & 2,2 \\
\hline C8:0 & 9,4 & 4,0 & $6,8+2,6$ & $1,6+0,6$ & 1,8 \\
\hline C10:0 & 11,3 & 4,0 & $13,8+3,5$ & $3,3+0,8$ & 3,6 \\
\hline C12:0 & 17,4 & 7,4 & $18,0+5,1$ & $4,3+1,2$ & 4,0 \\
\hline $\mathrm{C} 14: 0$ & 59,2 & 25,8 & $48,7+10,7$ & $11,6+2,5$ & 13,0 \\
\hline C15:0 & - & - & $6,3+2,2$ & $1,5+0,5$ & - \\
\hline C16:0 & 172,7 & 72,1 & $117,4+26,2$ & $27,9+6,2$ & 30,2 \\
\hline C16:1 & _- & - & $17,1+1,5$ & $4,1+0,4$ & - \\
\hline C17:0 & - & - & $6,3+1,6$ & $1,5+0,4$ & 13,7 \\
\hline C18:0 & 62,9 & 19,3 & $36,5+11,6$ & $8,7+2,8$ & 21,1 \\
\hline C18:1 & 178,0 & 48,1 & $118,4+35,5$ & $28,1+8,4$ & \\
\hline C18:2 & 25,8 & 4,7 & $19,0+7,2$ & $4,5+1,7$ & 3,0 \\
\hline C18:3 & 17,5 & 6,2 & $10,2+4,1$ & $2,4+1,0)$ & \\
\hline
\end{tabular}

$1=$ Composition en acides gras libres de deux emmentals suisses (Langler et Day, 1966).

2 = Composition en acides gras libres (moyenne, écart-type) des 54 emmentals de l'étude.

$3=$ Composition en acides gras totaux du lait de vache (Kuzdzal-Savoie et Kuzdzal, 1963). 
L'intensité moyenne de la protéolyse de l'Emmental "Grand-Cru" présente de grandes similitudes avec les résultats obtenus par Collin et al (1987) sur fromage de Comté. Dans les cas respectifs de ces deux fromages, les pourcentages moyens des fractions caséines $\beta(22,7$ et $18,7 \%)$, $\gamma_{1}+\gamma_{2}+\gamma_{3}(24,4$ et $23,4 \%), \alpha S_{1}(11,1$ et $13,8 \%), \alpha S_{1} \mid(35,0$ et $28,7 \%)$ sont assez voisins. Pour ces 2 produits fabriqués au lait cru, les similitudes de la protéolyse primaire pourraient être en partie attribuées à la nature des couples durée-température d'affinage; en l'occurence une durée plus courte ( 3 mois) et une température plus élevée $\left(21-23{ }^{\circ} \mathrm{C}\right)$ pour l'Emmental "Grand-Cru", contre une durée plus longue (4 à 9 mois) et une température plus basse $\left(18\right.$ à $\left.10^{\circ} \mathrm{C}\right)$ pour le gruyère de Comté.

La teneur en sel plus élevée dans le cas des fromages du groupe 3 (tableau IA) peut aussi contribuer à leur protéolyse plus faible (tableau IC).

La protéolyse plus importante, à âge égal, de l'Emmental Est-Central «GrandCru" (tableau II et fig 1), correspond très bien aux observations de Gallman (1982) qui démontrait l'existence d'une protéolyse plus intense dans le cas du fromage à raclette fabriqué au lait cru plutôt qu'au lait thermisé. En effet, le lait cru de grand mélange possède un système enzymatique protéolytique complexe, incluant les protéases endogènes (Humbert, 1986) ou exogènes du lait (Choisy et al, 1987). Humbert (1986) a montré que ce type de lait présente une forte activité endopeptidasique, pour une activité exopeptidasique plus limitée.

La thermisation pourrait ralentir la protéolyse des fromages en modifiant la flore et les systèmes protéolytiques du lait. La diminution de la flore totale des laits après thermisation a déjà été montrée (Coghill et al, 1982; Van den Berg, 1984) et il en résulte probablement une sélection de la flore présente dans les fromages.

L'étape de maturation avant fabrication des laits non thermisés ( 30 à $40 \mathrm{~min}$ à environ $32{ }^{\circ} \mathrm{C}$ ), pourrait aussi provoquer une augmentation de la flore naturelle du lait et accroître la teneur en protéases microbiennes effectivement actives dans le fromage.

L'analyse discriminante a montré la possibilité de caractériser l'Emmental EstCentral "Grand-Cru", à partir d'un nombre restreint de paramètres : NS/NT, $\mathrm{C}_{4}, \mathrm{iC}_{5}$, et $\mathrm{C}_{2}$. Toutefois, cette caractérisation présente des limites dans le cas où les fromages des autres types de fabrication ne développent pas de fermentation butyrique et peuvent en conséquence être affinés plus longtemps et présenter une protéolyse importante.

\section{CONCLUSION}

L'ensemble des précautions déployées lors de la production du lait et pendant la fabrication de l'Emmental Est-Central "Grand-Cru" conduit à l'obtention d'un produit typé, caractérisable sur le plan physico-chimique.

Ces fromages se distinguent tout au long de l'année des autres emmentals fabriqués en France par un profil fermentaire typiquement propionique, une lipolyse réduite et une protéolyse intense.

L'absence de fermentation butyrique et l'existence d'une lipolyse plus réduite dans les fromages d'Emmental Est-Central "Grand-Cru" sont des points importants qui permettent à la fois un affinage plus long et une meilleure conservation. 


\section{REMERCIEMENTS}

Ce programme a reçu une aide financière de la Région Franche-Comté (Contrat 3202 A). Nous remercions également Michel Courroye et Corinne Lachaux du laboratoire d'analyses de I'Institut Technique du Gruyère à Rioz (70240) pour leur contribution à la partie analytique de ce travail.

\section{RÉFÉRENCES}

Agnet $Y$ (1979) Dosage des acides gras volatils libres du fromage. Note technique non publiée : Laboratoire Départemental d'Analyses Agricoles, 39800 Poligny

Amariglio S (1986) Contrôle de la qualité des produits laitiers. Recueil de normes françaises AFNOR-ITSV, 227-228. Tour Europe, Cedex 07, 92080 Paris-La Défense

Aston JW, Creamer LK (1986) Contribution of the components of the water-soluble fraction to the flavour of Cheddar cheese. NZJ Dairy Sci Technol 21, 229-248

Berdagué JL, Jeunet R, Grappin R (1987) Affinage et qualité du Gruyère de Comté : III : Fermentation lactique et teneur en acides gras volatils des fromages de Comté. Lait 67, 249-263

Booy CJ (1968) Determination of salt content of cheese. Off Org K Ned Zuivelbond 60, 188189

Choisy C, Desmazeaud M, Gripon JC, Lamberet G, Lenoir J, Tourneur C (1987) Les phénomènes microbiologiques et enzymatiques et la biochimie de l'affinage. Dans : Le Fromage, (Eck A, ed), Technique et Documentation, Paris, pp 62-100

Cogan TM (1977) Heat resistant lipases and proteinases and the quality of dairy products. Int Standard FIL-IDF 43, 25-31

Coghill D, Mutzelburg ID, Birch SJ (1982) Effect of thermization on the bacteriological and chemical quality of milk. Aust J Dairy Technol $37,48-50$

Collin JC, Berdagué JL, Dognin-Bergeret $M$, Grappin R (1987) Affinage et qualité du Gruyère de Comté : IV. Etude de la protéolyse. Lait 67, 299-318
De Man JM (1966) Partial glycerides in the fat of Cheddar cheese. J Dairy Sci 49, 343-345

Driessen FM (1983) Lipases et proteinases in milk. Occurrence, heat inactivation, and their importance for the keeping quality of milk products. Neth Milk Dairy J 37, 193-196

Driessen FM, Stadhouders J (1975) Lipolysis in hard cheese made from pasteurized milk. Ann Bull Fed Int Lait 86, 101-103

Dumont JP, Adda J (1978) Flavour formation in dairy products. Flavour Research, Symposium Weurman, pp 245-262

FIL-IDF (1982) Fromages et fromages fondus. Détermination de l'extrait sec total. 4 A, 1-2

FIL-IDF (1986) Determination of nitrogen content (Kjeldahl method) and calculation of crude protein content. $20 \mathrm{~A}, 1-3$

FNPL-ITG (1982) Les butyriques, synthèse bibliographique. Fédération Nationale des Producteurs de Lait, 149 rue de Bercy 75595 Paris

Gallman P (1982) Einfluss der Rohmilchflora auf die biochemischen Vorgänge der Käsereifung am Beispiel von Raclette-Käse aus pasteurisierter und roher Milch. Thèse von Eidgenössischen Technischen Hochschule. N. 6972, Zurich

Heiss E (1961) Essais de dosage de la matière grasse dans le fromage par des méthodes rapides. Dtsch Molk Ztg 82, 67-70

Humbert G (1986) La protéase alcaline (plasmine) du lait : dosage, purification et implications en technologie laitière. Thèse de Doctorat ès Sciences, Université de Nancy I, pp $137-140$

Kuchroo CN, Fox PF (1982a) Fractionation of the water soluble nitrogen from Cheddar cheese: Chemical methods. Milchwissenschaft 37, 651-653

Kuchroo CN, Fox PF (1982b) Soluble nitrogen in Cheddar cheese : comparison of extraction procedures. Milchwissenschaft 37, 331-335

Kuzdzal-Savoie S, Day EA (1966) Contribution à l'étude de la lipolyse dans les fromages. Tech. Lait 14, 17-20

Kuzdzal-Savoie S, Kuzdzal W (1963) Contribution à l'étude des acides gras du lait de différentes espèces animales (2). Lait 43, 369384 
Kuzdzal-Savoie S, Kuzdzal W (1967) Les acides gras libres du fromage. Lait 47, 9-23

Langler JE, Day EA (1966) Quantitative analyses of the major free fatty acids in Swiss cheese. J Dairy Sci 49, 91-93

Lenoir J (1963) Note sur la dégradation des protides au cours de la dégradation du Camembert. Lait 43, 154-165
Pearce KN (1977) The complexometric determination of calcium in dairy products. NZJ Dairy Sci Technol 12, 113-115

Supelco (1975) Fatty acid analysis. Supelco, Inc. Bellefonte, PA 16823, 1-4

Van den Berg MG (1984) The thermization of milk. Bull Fed Int Lait 182, 3-11 